\title{
Object-based selection under focused attention: A failure to replicate
}

\author{
DOMINIQUE LAMY \\ Tel Aviv University, Tel Aviv, Israel
}

\begin{abstract}
In a recent study, Lavie and Driver (1996) reported that object-based effects found with distributed attention disappear when attention is focused on a narrow area of the display. This finding stands in contrast with previous reports of object-based effects under conditions of focused attention (e.g., Atchley \& Kramer, 1998; Egly, Driver, \& Rafal, 1994). The present study was an attempt to replicate Lavie and Driver's finding, using similar task and stimuli. While Lavie and Driver's object-based effect in the distributed attention condition was replicated, its absence in the focused attention condition was not. In the two experiments reported in this paper, object-based effects were found under conditions of both distributed and focused attention, with no difference in the magnitude of the object-based effects in the two conditions. It is concluded that, in contrast with Lavie and Driver's claim, the initial spatial setting of attention does not influence object-based constraints on the distribution of attention.
\end{abstract}

A central issue in the study of visual selective attention concerns the representational format in which selection takes place. In the last 15 years, numerous studies have investigated whether attentional selection operates within space-based or within object-based representations (see Egeth \& Yantis, 1997, for a review).

Evidence coming from a wide range of paradigms shows that the distribution of attentional resources is constrained by grouping factors other than proximity, thus providing strong support for the object-based view. Using the Eriksen response competition paradigm or flanker task (Eriksen \& Hoffman, 1973), several experiments showed that distractors slow response to a target more when they are grouped with it (e.g., by common color or contour) than when they are not (e.g., Baylis \& Driver, 1992; Driver \& Baylis, 1989; but see Berry \& Klein, 1993, and Kramer, Tham, \& Yeh, 1991, for failures to replicate; see also Kramer \& Jacobson, 1991). Other studies showed that, after subjects have been cued to attend to a location within a certain object, it is easier for them to reorient their attention to an invalid location in the same object than to an invalid location in a different object (e.g., Egly, Driver, \& Rafal, 1994; Vecera, 1994). Finally, other authors showed that it is easier to divide attention between two aspects of the display that belong to the same object rather than to different objects (e.g., Duncan, 1984; Kramer, Weber, \& Watson, 1997; Treisman, Kahneman, \& Burkell, 1983; Vecera \& Farah, 1994; Watson \& Kramer, 1999).

This research was supported by the Israel Science Foundation and by the Israel Foundations Trustees. The author thanks Steven Yantis, Art Kramer, Zhe Chen, Nilli Lavie, Jon Driver, and two anonymous reviewers for useful comments. Correspondence should be addressed to D. Lamy, Department of Psychology, Johns Hopkins University, Baltimore, MD 21218 (e-mail: domi@www.psy.jhu.edu).
Lavie and Driver (1996, p. 1239) noted that "most reports of object-based limits to divided visual attention have stemmed from situations where the spatial separation between judged attributes was held constant and at a minimum level" (e.g., Duncan, 1984). On the basis of this observation, they suggested that "object-based factors may only become important within a hypothetical 'spotlight' or 'zoom-lens' of spatial attention, when no further spatial restriction is possible" (Lavie \& Driver, 1996, p. 1240).

In order to investigate whether object factors dominate only within narrow displays, Lavie and Driver (1996) used displays in which subjects had to divide their attention over large distances, with a procedure including several improvements over previous reports of object-based selection under conditions of divided (or distributed) attention. ${ }^{1}$ Stimulus displays consisted of two long intersecting dashed lines. Each line had a different color and comprised two odd elements, a white dash or a white dot (Experiments 3 and 4). Subjects had to judge whether the two odd elements were the same or different-that is, whether there were two white dashes or white dots (same response) or a white dash and a white dot (different response). The odd elements were either close to each other on different lines (near condition) or far apart by approximately $8^{\circ}$ of visual angle. When far apart, they belonged either to the same line (object condition) or to different lines ( far condition). The results showed better performance in the object condition than in the far condition, revealing object-based constraints on the distribution of attention (Experiments 1-3). Thus, these results indicate that previously reported object-based effects on dividing attention may also be found when the display covers a very large extent. Lavie and Driver noted that this finding does not necessarily preclude the possibility that object-based selection operates only within spatially attended area, because subjects may have distributed their attention across 
the display, "giving their attentional 'zoom lens' . . a wide initial setting" (Lavie \& Driver, 1996, p. 1246).

Lavie and Driver's (1996) fourth experiment was designed to test whether object-based effects would still be found when subjects adopted a narrow focus of attention. A precue indicated the side of the display in which both targets were most likely to appear. This manipulation yielded four conditions: valid-near ( $70 \%$ of the trials), invalid-near $(10 \%$ of the trials), invalid-object $(10 \%$ of the trials), invalid-far ( $10 \%$ of the trials). The critical comparison, between the last two conditions, showed that when subjects focused their attention narrowly within the display, object-based effects disappeared (i.e., the invalidobject condition was not faster than the invalid-far condition).

Lavie and Driver (1996) concluded that the initial spatial setting of attention may influence object-based constraints on the distribution of attention. Namely, they proposed that object-based effects may be found when attention is distributed in a diffuse mode across the display, but not when it is focused on a narrow spatial area within this display.

Such results are important, since they appear to uncover new boundary conditions between space-based and object-based selection. They have also influenced the rationale of several recent studies (e.g., Atchley \& Kramer, 1998; Neely, Dagenbach, Thompson, \& Carr, 1998). However, Lavie and Driver's (1996) last finding (Experiment 4) and the resulting conclusions are surprising, because they are at odds with other reports in the literature. Indeed, object-based effects were obtained in studies in which attention was focused on a small part of the display's objects (e.g., Atchley \& Kramer, 1998; Baylis \& Driver, 1992; Driver \& Baylis, 1989; Egly et al., 1994; Kramer \& Jacobson, 1991).

Sometimes, the distance between the relevant parts of the perceptual groups for which object-based effects were measured was very small (e.g., $1.2^{\circ}$ of visual angle in Baylis \& Driver, 1992, and in Driver \& Baylis, $1989 ; 0.25^{\circ}-1^{\circ}$ of visual angle in Kramer \& Jacobson, 1991). Thus, following Lavie and Driver's argument, one may assume that, in these studies, attention could not be restricted to the cued location and was in fact distributed over the whole region occupied by the objects. It is therefore not surprising that object-based effects were obtained.

Other findings, however, do pose a more potent threat to Lavie and Driver's idea that no object-based effects are found within large displays when attention is tightly focused on a narrow location.

Egly et al. (1994) had their subjects detect a luminance change in one of the four corners of two outline rectangles. One corner was precued. On valid-cue trials, the target appeared in the cued corner of the cued rectangle, whereas on invalid-cue trials, it appeared either in the noncued corner of the cued rectangle or in the uncued rectangle. The distance between the cued location and the location where the target actually appeared was identical in both invalid-cue conditions. Egly et al. found the cost of redirecting attention to an invalid location to be greater for targets in the noncued rectangle than in the cued rectangle, indicating the presence of object-based effects. These results have been replicated several times (e.g., Macquistan, 1997; Neely \& Dagenbach, 1997; Vecera, 1994). Thus, in this study, object-based effects were found under conditions in which, according to Lavie and Driver's conclusion, space-based selection should have prevailed. Namely, attention was focused on a narrow area by a precue, and the relevant objects, which extended outside this narrow area, were comparable in size to the objects used by Lavie and Driver $\left(11.4^{\circ}\right.$ vs. $12.6^{\circ}$ or $13^{\circ}$, respectively).

Atchley and Kramer (1998) conducted a series of experiments using a more realistic version of Egly et al.'s (1994) stimuli-namely, stimuli that transcended depth and resembled pipes attached to a surface. These stimuli were large: The length of the pipes varied between $13.6^{\circ}$ and $16.3^{\circ}$ of visual angle. Subjects were required to perform a detection task. They had to judge whether one or two white dots ("leaks" on the pipes) were present in the display. When there were two targets, they could appear on the same or on different pipes. Atchley and Kramer found that subjects were significantly faster in the sameobject condition (Experiment 1). Most importantly, and in contradiction with Lavie and Driver's hypothesis, this object-based effect was not reduced (it was actually larger) when a spatial precue summoned attention to a small region of the display (Experiments 2 and 3 ).

There are several differences between Egly et al.'s (1994) and Atchley and Kramer's (1998) studies, and Lavie and Driver's (1996) study. In Egly et al.'s study, the objects were presented before the cue appeared, thus providing subjects with the opportunity to segment the visual field before attention was focused by the cue. In contrast, in Lavie and Driver's study, the cue appeared in an otherwise empty field, the objects being presented only after cue offset. However, this difference is unlikely to be the source of the conflicting results, because in Atchley and Kramer's study, the cue also preceded object presentation, and object-based effects were nonetheless obtained.

An additional difference is that they used different tasks. Whereas the former required subjects to perform a detection task, the latter required them to perform a discrimination task. However, task type is unlikely to account for the discrepancy between the results of these studies because other authors obtained object-based effects under similar conditions, using discrimination tasks (e.g., Chen, 1998; Moore, Yantis, \& Vaughan, 1998).

The results obtained by Neely et al. (1998) suggested a potential role for the stimulus onset asynchrony (SOA) between the cue used to focus attention on a small area and the target display in which object-based effects are measured for different cue-to-location distances. Neely et al. reported object-based effects for far cue-to-target distances at short SOAs $(100 \mathrm{msec})$, when attention was presumably still distributed over a large portion of the 
display. In contrast, they found these effects to be eliminated at longer SOAs, at which attention was likely to be more focused on the cued location. These findings are consistent with Lavie and Driver's idea that object-based effects may occur only within the spatial extent of attention. However, in follow-up studies, Neely et al. failed to replicate their finding of an object-based effect using the short SOA. ${ }^{2}$ Still, although further research is needed in order to assess the role of SOAs in object-based selection, it may be useful to look into SOA differences between Lavie and Driver's (1996) study and other studies. Lavie and Driver used a 70-msec cue-to-target SOA and found no object-based effect, whereas Egly et al. (1994) and Atchley and Kramer (1998) used 300- and 100-msec SOAs, respectively, and did find object-based effects. The difference between 70 - and 100 -msec SOAs is very small, so there is only a frail possibility that SOA differences may account for the discrepant results.

A last difference worth noting is that in Atchley and Kramer's (1998) and Egly et al.'s (1994) experiments, the cue occupied a location neighboring or belonging to, respectively, only one of the two objects present in the display. Lavie and Driver (1996) used a cue consisting of two elements, each of which appeared at a location subsequently occupied by different objects. Thus, one may argue that, in the former studies, the object was cued along with the cued location, which may explain why objectbased effects were found. In contrast, in the latter study, the cue did not direct attention toward a particular object, only toward a particular location, which may explain why only space-based effects were obtained.

Before engaging in a systematic investigation of the role of the differences just listed, an attempt was made to replicate Lavie and Driver's (1996) results. Again, their most consequential finding was the absence of any objectbased effect under conditions of focused attention. Except for three minor differences in the procedure, the first experiment presented below was identical to Lavie and Driver's Experiment 4, in which attention was focused on just one side of the display. These differences were the following: (1) The actual size of the stimuli used in the present experiment was slightly smaller than in Lavie and Driver's. (2) Subjects responded with the index or middle finger to signal that the targets were the same or different, respectively, whereas in Lavie and Driver's experiment, they responded with the thumb or index finger. (3) The present experiment was shorter. It included 50 practice trials and 500 experimental trials, whereas Lavie and Driver's experiment included 80 practice trials and 800 experimental trials.

\section{EXPERIMENT 1}

\section{Method}

Subjects. The subjects were 12 Tel Aviv University undergraduates who participated in the experiment for course credit. All reported having normal or corrected visual acuity and normal color vision.

Apparatus. Displays were generated by an IBM PC-compatible computer attached to a Super VGA color monitor, using $800 \times 600$ graphics mode. Responses were collected via the computer keyboard. The experiment was written and run using a $\mathrm{C}++$ program.

Stimuli. The fixation display was a white $0.1^{\circ} \times 0.1^{\circ}$ plus sign $(+)$ in the center of a gray background $[\operatorname{RGB}=(53,53,53)$, and $\left.\operatorname{lum}=3 \mathrm{~cd} / \mathrm{m}^{2}\right]$.

The target display consisted of two straight dashed lines intersecting at their midpoint in the center of the screen. One line was horizontal, and the other was tilted $18^{\circ}$ clockwise from the horizontal (see Figure 1). In each display, one line was pink [RGB = $(213,0,213)$, and lum $\left.=18 \mathrm{~cd} / \mathrm{m}^{2}\right]$, and the other was yellow

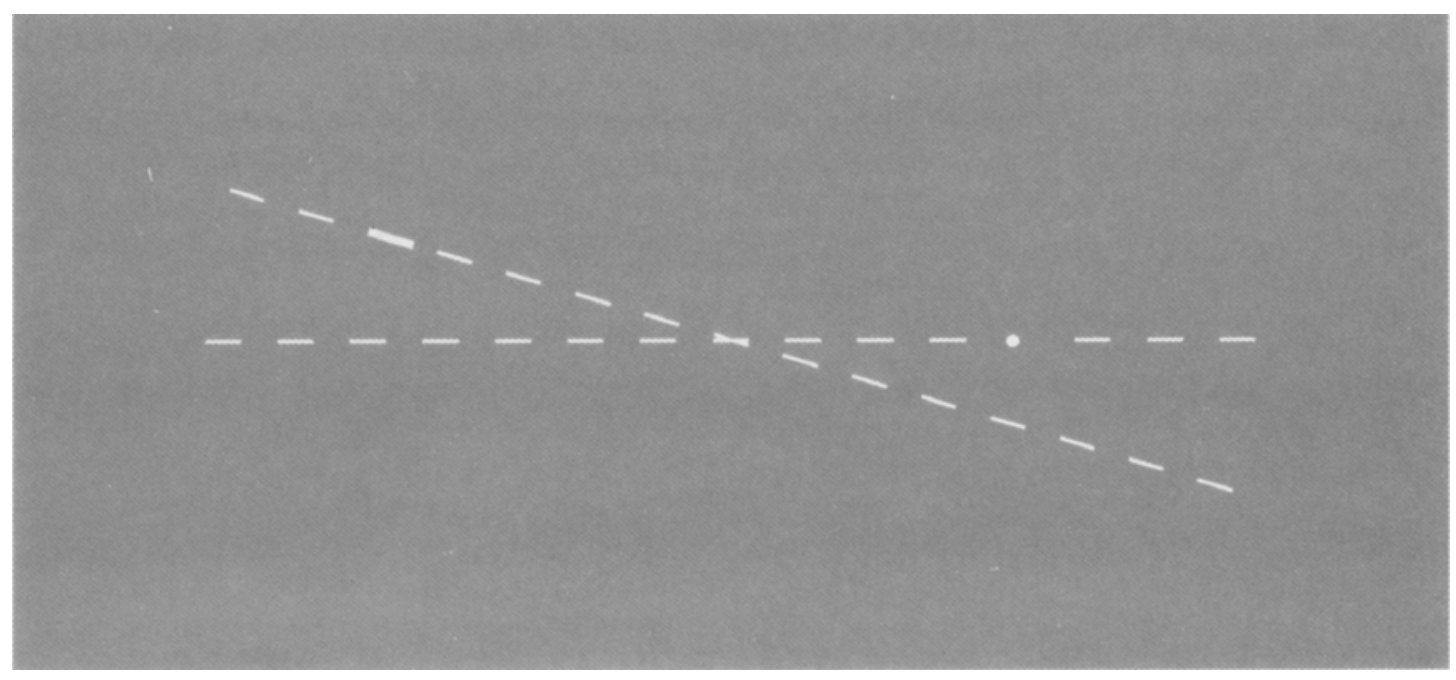

Figure 1. Sample stimulus display for the far condition (the two targets are far from each other on different lines), requiring a different judgment (the two targets are different from each other). The bold elements represent the white targets. The lines were presented in different colors (pink and yellow), and the background was gray. 
$\left[R G B=(213,213,0)\right.$, and lum $\left.=59 \mathrm{~cd} / \mathrm{m}^{2}\right]$. The horizontal and tilted lines were equally likely to be yellow or pink. They subtended $12.6^{\circ}$ and $13^{\circ}$ of visual angle $(13.2$ and $13.7 \mathrm{~cm})$, respectively. Each line was made up of 15 evenly spaced dashes. Each dash subtended $0.6^{\circ}$ in length and 2 pixels in height. The distance between adjacent dashes was $0.32^{\circ}$ and $0.37^{\circ}(0.34$ and $0.39 \mathrm{~cm})$ for the horizontal line and the tilted line, respectively.

Each display contained two targets, which replaced two of the colored dashes. There were two possible types of target: a white a dot or a white dash. The target dot subtended $0.2^{\circ}$ in diameter and was centered on the center of the location of the dash that it replaced. The target dash was identical to the dash that it replaced except that it was white instead of colored. One of the targets replaced the third dash from the end of a line, and the other target replaced the fourth dash. Thus, the two targets always appeared in the periphery of the display. They were equally likely to replace the third or fourth dash from the end of the horizontal or tilted line.

In each display, the two targets were equally likely to be the same or different and, when the same, to be both white dots or white dashes. They were also equally likely to appear in the horizontal or in the vertical line. Finally, there were three possible pairs of locations for the two targets (see Figure 1): at opposite ends of the same line (object condition); at opposite ends of the display on different lines ( far condition); on the same end of the display on different lines (near condition). The center-to-center distance between the two targets was $8.2^{\circ}(8.6 \mathrm{~cm})$ in the object condition, $7.8^{\circ}(8 \mathrm{~cm})$ in the far condition, and $1.3^{\circ}$ or $1.6^{\circ}(1.4$ or $1.7 \mathrm{~cm})$ in the near condition.

The cuing display consisted of the endmost colored dashes of both lines on either the left or the right side of the display. These segments were also present in the target display, which followed the cuing display. Their earlier onset caused the display to flicker on the cued side.

Procedure. The subject sat approximately $60 \mathrm{~cm}$ from the monitor in a darkened room, with his/her head on a chinrest. Each trial began with the fixation display, which remained on the screen for $1 \mathrm{sec}$. The cuing display immediately followed and appeared for $70 \mathrm{msec}$. It was replaced by the target display, which appeared for $130 \mathrm{msec}$. The screen went blank until the subject responded. ${ }^{3}$ The cue was valid on $70 \%$ of the trials. That is, on $70 \%$ of the trials, the two targets had the near arrangement and appeared in different lines on the cued side of the display (valid-near condition). In the remaining trials, the targets were equally likely to appear on the uncued side of the display (invalid-near condition, $10 \%$ of the trials), on distinct lines on opposite sides of the display (invalid-far condition, $10 \%$ of the trials), or at the opposite ends of the same line (invalid-object condition, $10 \%$ of the trials).

Same and different responses were required equally often for each of the four conditions. Within each condition, the cued side was equally likely to be on the left or on the right. The subjects were given 50 practice trials randomly selected from the experimental trials, preceded by a short block of 10 examples, which the subjects simply watched. There were five blocks of 100 experimental trials. The subjects were allowed a rest period between them. Thus, this experiment was shorter than the original experiment by Lavie and
Driver (1996), since it included 500 experimental trials instead of 800 ( 10 blocks of 80 trials each).

The subjects were told that two dashed lines crossing each other would appear on each trial, one pink and the other yellow. They were informed that two of the dashes making up the lines would be replaced by two odd elements, either a white dot or a white dash. They were also told that the odd elements, the targets, would appear at the periphery of the display, in all possible pairwise combinations of the four ends of the lines. They were instructed to judge whether the two targets were the same (two white dots or two white dashes) or different (one white dot and one white dash). The subject responded with the right hand, using the numerical keypad on the right side of the standard computer keyboard, pressing " 0 " with the index finger to indicate a same judgment and " 2 " with the middle finger to indicate a different judgment. ${ }^{4}$ The subjects were informed that, on each trial, the target display would be preceded by a cue on one side of the display, which would convey the impression that the display flickered on the cued side of the display. They were instructed to pay attention to the cued side of the display, because, on most of the trials, the two targets would appear on that side of the display. However, they were also told that, on a small proportion of the trials, at least one target and possibly both would appear on the uncued side. It was emphasized that they should respond as fast as possible while minimizing the number of errors. A $500-\mathrm{msec}$ feedback tone was immediately sounded after error responses.

\section{Results}

Table 1 shows the average reaction times (RTs) and accuracy rates for each of the four conditions, for the two response types. Trials with RTs exceeding each subject's mean RT by more than 3 standard deviations were excluded from analysis. This removed $1.9 \%$ of the correct responses across subjects.

In order to determine whether or not the cuing procedure was effective in directing the subjects' attention to the cued side of the display, RT data from the invalidnear, invalid-object, and invalid-far conditions were pooled for comparison with the valid-near condition. A two-way analysis of variance (ANOVA) of the RT data on correct trials [validity $(2) \times$ response $(2)$ ] revealed a main effect of validity $[F(1,11)=16.71, p<.002]$, with faster responses in the valid-near condition. There was also a main effect of response $[F(1,11)=7.98, p<.02]$, with faster same responses. The interaction between the two factors was not significant $(F<1)$. A planned contrast showed that the valid-near condition was significantly faster than the invalid-near condition $[F(1,11)=11.85, p<.006]$.

An analysis comparing RTs in the three invalid-cue conditions revealed an effect of condition $[F(2,22)=3.98$, $p<.04$ ], which did not interact with response $(F<1)$.

Table 1

Mean RTs (in Milliseconds) and Accuracy Rates (\%) Across Subjects $(n=12)$ as a Function of Condition and Response in Experiment 1

\begin{tabular}{|c|c|c|c|c|c|c|c|c|}
\hline \multirow[b]{3}{*}{ Response } & & & \multicolumn{6}{|c|}{ Invalid Condition } \\
\hline & \multicolumn{2}{|c|}{ Valid-Near Condition } & \multicolumn{2}{|c|}{ Near } & \multicolumn{2}{|c|}{ Object } & \multicolumn{2}{|c|}{ Far } \\
\hline & RT & $\%$ & RT & $\%$ & RT & $\%$ & RT & $\%$ \\
\hline Same & 599 & 91 & 630 & 93 & 630 & 93 & 637 & 88 \\
\hline Different & 631 & 91 & 654 & 92 & 650 & 91 & 688 & 88 \\
\hline
\end{tabular}


RTs were significantly faster in the invalid-near condition than in the invalid-far condition $[F(1,11)=5.93$, $p<.04]$. This effect did not interact with response $(F<$ 1). Most importantly, RTs in the invalid-object condition were significantly faster than in the invalid-far condition $[F(1,11)=5.60, p<.04]$, revealing the presence of an object-based effect. The interaction of the object-based effect with response did not reach significance $[F(1,11)=$ $3.62, p<.09$ ].

Comparable analyses were conducted on the accuracy data. A two-way ANOVA [validity $(2) \times$ response $(2)$ ] showed no effect of validity $(F<1)$ and no effect of response $(F<1)$. The two factors did not interact $(F<1)$. A planned contrast between the valid-near condition and the invalid-near condition showed that the subjects tended to be more accurate when the two targets were on the cued side of the display than on the uncued side $[F(1,11)=$ $3.93, p<.08]$. A comparison between the three invalid conditions revealed an effect of condition $[F(2,22)=5.22$, $p<.02]$. A planned contrast revealed that performance was significantly better in the invalid-near condition than the invalid-far condition $[F(1,11)=16.09, p<.002]$. The most important comparison, between the invalid-object and invalid-far conditions, showed the same pattern as for the RT data. Namely, the subjects were more accurate in the invalid-object condition than in the invalid-far condition $[F(1,11)=4.98, p<.05]$. This result rules out any speed-accuracy tradeoff as an explanation for the objectbased effect.

\section{Discussion}

The results of Experiment 1 clearly reveal an objectbased effect, which was significant in both the RT data and the accuracy data. Thus, although the task and stimuli used in this experiment were similar to those used in Lavie and Driver's (1996) Experiment 4, their most consequential finding - namely, the absence of an objectbased effect under conditions in which attention is focused on a small area within a large display-was not replicated. In contrast, all the other findings of their experiment were replicated. First, the cuing procedure was efficient in directing attention toward one side of the display. Indeed, the main effect of validity and the difference between the valid-near and invalid-near conditions were significant. These effects were comparable to Lavie and Driver's in magnitude and statistical significance (main effect of validity, 33 vs. $59 \mathrm{msec}, p<.002$ vs. $p<.001$; valid-near vs. invalid-near, 27 vs. $26 \mathrm{msec}, p<.006$ vs. $p<.03)$. Thus, it is unlikely that the object-based effects obtained here stemmed from the fact that attention was insufficiently focused. Second, the main effect of response was replicated, which suggests that the difference between the two experiments concerning which fingers were used to respond was inconsequential. Finally, the faster performance in the near-invalid condition relative to the farinvalid condition was also replicated.

Finding object-based effects under conditions of focused attention does not necessarily undermine the gen- eral idea that the initial spatial setting of attention affects object-based constraints on attention. Indeed, this idea may still prove valid if object-based effects are found to be significantly larger with distributed attention than with focused attention. Following this rationale, one may argue that the present apparatus and stimuli were slightly different from Lavie and Driver's and, as a result, yielded larger object-based effects.

In order to examine this possibility, a control group of 11 new subjects was run on a distributed-attention condition. This condition was a replication of Lavie and Driver's (1996) Experiment 3 using the present apparatus and stimuli, except for the same minor changes as in the focused-attention condition, concerning the events following stimulus offset, finger-to-response mapping, and experiment length. It was identical to the focused attention condition except for the following changes. There was no cuing display. The target display immediately followed the fixation display and appeared for $177 \mathrm{msec}$, instead of only $130 \mathrm{msec}$. Attention was therefore distributed across the display, and there were three conditions instead of four: the object condition ( $25 \%$ of the trials), the far condition ( $25 \%$ of the trials), and the near condition ( $50 \%$ of the trials). The main findings of Lavie and Driver's Experiment 3 were replicated. In particular, RTs in the object condition were faster than those in the far condition $[F(1,10)=6.58, p<.03]$, indicating the presence of an object-based effect, which did not interact with response $(F<1)$. However, and most critically, this effect was comparable to the object-based effect obtained in the focused-attention condition ( $23 \mathrm{vs.} 10 \mathrm{msec} ; F<1$ ).

Thus, taken together, the results obtained in the present experiment for the focused-attention and distributedattention conditions strongly suggest that the initial spatial setting of attention-namely, whether attention is in a distributed mode or in a focused mode - does not affect object-based constraints on the distribution of attention.

\section{EXPERIMENT 2}

Lavie and Driver's (1996) subjects completed 800 experimental trials, whereas in Experiment 1 reported here, subjects were run on only 500 experimental trials. Thus, Lavie and Driver's subjects received substantially more practice than did the subjects in Experiment 1 of the present study. This difference opens the possibility that objectbased effects may decrease with practice, which could explain the discrepancy between the two studies. ${ }^{5}$ Weaver, Lupiáñez, and Watson (1998) reported a similar phenomenon, by showing that object-based inhibition of return decreases with practice.

Moreover, Lavie and Driver (1996) did not specify the exact coordinates they used for the background's gray color and for the lines' pink and yellow colors. Thus, the specific colors used in Experiment 1 may have slightly differed from theirs. The two lines were more luminant than the background and thus stood out strongly against it. The difference in luminance between the lines and 
background was larger than between the lines and targets, which conveyed the impression that the targets were embedded in the lines rather than popping out. These differences may have been smaller in Lavie and Driver's displays, thus minimizing object-based effects. Note, however, that only the attempt to replicate the absence of object-based effects with focused attention failed. The presence of these effects with distributed attention, in contrast, was replicated, using the same stimuli. Thus, in order to account for the pattern of results found here, one must make the additional assumption that this difference in relative luminance affected performance more under conditions of focused attention than under conditions of distributed attention. That is, one must assume that more "objectness" is required to yield object-based effects with focused attention than with distributed attention.

Experiment 2 was conducted in order to test the role of practice and of potential differences in stimulus colors, in accounting for the discrepancy between the results of Lavie and Driver's (1996) Experiment 4 and the results of Experiment 1 of the present study. Accordingly, Experiment 2 included 80 practice trials and 800 experimental trials, exactly as in Lavie and Driver's experiment. Moreover, the luminance difference between the background and lines was significantly reduced. If the relative salience of the lines was responsible for the failure to replicate Lavie and Driver's finding in Experiment 1 , the object-based effect found in that experiment should disappear with the new stimuli. Moreover, because replicating the present object-based effects with stimuli that matched Lavie and Driver's description but differed as much as possible from the stimuli of Experiment 1 would strengthen the generality of the present finding, different hues of yellow and pink for the lines were also used. Finally, in an effort to further reduce the differences between Lavie and Driver's and the present study, three additional changes were introduced. First, the same number of subjects participated in the present experiment - that is, 20 subjects, instead of only 12 as in Experiment 1. Second, the same finger-to-response mapping was used as in Lavie and Driver's study. Third, the results were analyzed using the same procedure to exclude outliers-namely, RTs exceeding $2,000 \mathrm{msec}$ were removed from the analysis, rather than RTs exceeding each subject's mean by more than 3 standard deviations (as in Experiment 1).

\section{Method}

Subjects. The subjects were 20 Tel Aviv University undergraduates who were paid $\$ 6$ for their participation. None of them had participated in Experiment 1. All reported having normal or corrected visual acuity and normal color vision.

Apparatus, Stimuli, and Procedure. The apparatus, stimuli, and procedure were the same as in Experiment 1, except for the following changes. First, the subjects were run on 80 practice trials, followed by 10 blocks of 80 experimental trials each. Second, the luminance difference between the background and line was reduced. The new color coordinates were $R G B=(150,150,150)$, and lum $=28 \mathrm{~cd} / \mathrm{m}^{2}$, for the gray background; $R G B=(255,128$, $255)$, and lum $=42 \mathrm{~cd} / \mathrm{m}^{2}$, for the pink line; and RGB $=(191,191$, 0 ), and lum $=47 \mathrm{~cd} / \mathrm{m}^{2}$, for the yellow line. Finally, in this experiment, the subjects responded with the same fingers as did Lavie and Driver's subjects: with the thumb to indicate same judgments and with the index finger to indicate different judgments.

\section{Results}

Table 2 shows the average RTs and accuracy rates for each of the four conditions, for the two response types. The data from 1 subject were excluded because this subject's error rate exceeded $35 \%$. Trials with RTs exceeding $2,000 \mathrm{msec}$ were excluded from analysis. This removed $0.6 \%$ of the correct responses across subjects.

The same analyses were conducted as in Experiment 1. A two-way ANOVA of the RT data on correct trials [validity (2) $\times$ response (2)] revealed a main effect of validity $[F(1,18)=20.90, p<.0002]$, with faster responses in the valid-near condition. There was also a main effect of response $[F(1,18)=4.60, p<.05]$, with faster same responses. The interaction between the two factors was not significant $(F<1)$. A planned contrast revealed faster RTs in the valid-near condition than in the invalidnear condition $[F(1,18)=11.70, p<.003]$.

An analysis comparing RTs in the three invalid-cue conditions revealed a significant effect of condition $[F(2,36)=5.30, p<.01]$, which did not interact with response $[F(2,36)=2.50, p=.10]$. RTs in the invalidnear condition were significantly faster than those in the invalid-far condition $[F(1,18)=9.49, p<.006]$. This effect interacted with response $[F(1,18)=4.70, p>.04]$, the advantage in the invalid-near condition being larger for different responses than for same responses. Again, and most importantly, RTs in the invalid-object condition were significantly faster than those in the invalidfar condition $[F(1,18)=5.62, p<.03]$. This effect did not interact with response $(F<1)$.

Table 2

Mean RTs (in Milliseconds) and Accuracy Rates (\%) Across Subjects $(n=19)$ as a Function of Condition and Response in Experiment 2

\begin{tabular}{|c|c|c|c|c|c|c|c|c|}
\hline \multirow[b]{3}{*}{ Response } & & & \multicolumn{6}{|c|}{ Invalid Condition } \\
\hline & \multicolumn{2}{|c|}{ Valid-Near Condition } & \multicolumn{2}{|c|}{ Near } & \multicolumn{2}{|c|}{ Object } & \multicolumn{2}{|c|}{ Far } \\
\hline & RT & $\%$ & RT & $\%$ & RT & $\%$ & $\mathrm{RT}$ & $\%$ \\
\hline Same & 525 & 96 & 544 & 95 & 547 & 95 & 566 & \\
\hline Different & 545 & 95 & 563 & 94 & 578 & 94 & 615 & \\
\hline
\end{tabular}


Comparable analyses were conducted on the accuracy data. A two-way ANOVA showed no effect of response $[F(1,18)=2.28, p>.1]$. There was a small tendency for valid trials to be more accurate than invalid trials $[F(1,18)=3.66, p<.08]$. Validity did not interact with response $(F<1)$. The planned comparisons conducted for the RT data were also conducted for the accuracy data. No effect reached significance. Thus, there was no speed-accuracy tradeoff.

\section{Discussion}

The present results basically replicated the findings of Experiment 1. First, and most crucially, the object-based effect found in Experiment 1 was replicated. Thus, in Experiment 2, a second failure to replicate Lavie and Driver's (1996) finding was reported. However, in order to conclude that the spatial extent of attention does not modulate object-based selection, it was again important to ensure that object-based effects would be found with the new stimuli under conditions of distributed attention and to compare their magnitude relative to the focused attention condition. To do that, a control group of new subjects was run on a distributed-attention condition. This condition was identical to the control condition run in Experiment 1 except for the changes in the colors and fingerto-response mapping used in the focused-attention condition of the present experiment. An analysis of the RT data showed that RTs in the object condition were faster than those in the far condition $[F(1,11)=5.72, p<.04]$. Most importantly, there was no difference between the magnitude of the object-based effect in the focused-and distributed-attention conditions using the new stimuli ( 28 vs. $18 \mathrm{msec} ; F<1$ ).

In contrast with this failure to replicate an absence of object-based effect, most of the other findings of Lavie and Driver's (1996) experiment were replicated. The cuing procedure was efficient, as indicated by the significant effect of validity and by the significant advantage on valid-near trials relative to invalid-near trials. There was an effect of response, with faster same responses. RTs in invalid-near trials were faster than those in invalid-far trials. The only departure from the pattern of results reported by Lavie and Driver and also from the results of Experiment 1 was the significant interaction between response and the near-invalid versus far-invalid effect, for which I have no plausible explanation.

These results suggest that the object-based effect found in Experiment 1 was relatively robust, since it was impervious to changes in objects' salience relative to the background and to changes in hues.

Moreover, they indicate that there was no interaction between practice and object-based effects. Indeed, objectbased effects of the same magnitude were found in Experiment 1, which included 500 trials, and in Experiment 2, which, as in Lavie and Driver's (1996) study, included 800 trials. This was further confirmed by an ANOVA with condition (invalid-near, invalid-object, invalid-far) and block (first half of the experiment, second half of the experiment) as factors. Although there was a highly significant main effect of block $[F(1,18)=19.67, p<.0003]$, with RTs being significantly reduced in the second half of the experiment relative to the first one ( $576 \mathrm{vs} .513 \mathrm{msec})$, the interaction between condition and block was nonsignificant $[F(2,36)=1.73, p>.2]$. In particular, a planned contrast revealed that the object-based effect (invalid-object vs. invalid-far conditions) did not interact with block $[F(1,18)=1.01, p>.3]$.

Thus, the results of the present experiment reinforce the finding of Experiment 1-namely, the reported failure to replicate Lavie and Driver's (1996) finding that object-based effects obtained under conditions of distributed attention disappear when attention is focused on a small area.

\section{GENERAL DISCUSSION}

The original motivation for our research was to examine which factors may account for the discrepancy between the results presented by Lavie and Driver (1996, Experiment 4) and those presented by Egly et al. (1994) and Atchley and Kramer (1998). The former found that object-based constraints on the distribution of attention that are revealed when attention is widely distributed do not affect performance when attention is focused on a small area. In contrast, the latter did find object-based effects when attention was focused by a precue on a narrow area within a large display (i.e., under conditions in which, according to Lavie and Driver's conclusion, spacebased selection should have prevailed). Several differences between the two sets of studies were pointed out. In particular, Lavie and Driver used a shorter cue-to-target SOA than did Egly et al. and Atchley and Kramer. Moreover, the former used a cue that pointed to a specific location in the visual display but not to a specific object, whereas the latter used a cue that pointed to both a specific location and a specific object. Before investigating the role of these factors, an attempt was made to replicate Lavie and Driver's findings.

Unfortunately, the results of the present experiments were quite disappointing. Two failures to replicate Lavie and Driver's (1996) finding that no object-based effects are found when attention is focused on a small area of the display were reported. Object-based effects were found under conditions of both distributed and focused attention, with no difference in the magnitude of the object-based effects in the two conditions. In order to reduce the differences between the two studies, and lest the differential amount of practice given to subjects may have accounted for the discrepancy in the results, the number of trials was increased from 500 to 800 , as in Lavie and Driver's experiment. Moreover, the number of subjects was equalized to Lavie and Driver's, as well as finger-to-response mapping. Finally, in order to minimize the probability that the reported failure to replicate may 
have stemmed from unintended differences in the displays used, a new set of stimulus colors was used, which differed in relative luminance and hue from that used in Experiment 1. A significant object-based effect was obtained despite these changes.

The fact that the same results were obtained over a range of stimuli reinforces their reliability. Moreover, it is noteworthy that these results are based on significant effects, and, as such, they have more statistical power than Lavie and Driver's (1996) results, which are based on a null effect. Finally, the present results are consistent with previous reports in the literature (e.g., Atchley \& Kramer, 1998; Egly et al., 1994), so that, in contrast with Lavie and Driver's findings, they do not require additional hypotheses as to how object-based constraints affect the distribution of attention. In light of the above, although the source of the difference between our results and those reported by Lavie and Driver remains unknown, it seems reasonable to assume that their finding is a fragile phenomenon that may be contingent on subtle display differences and that should be regarded as an exception rather than as the rule.

To conclude, the results of the experiments presented here strongly support the idea that, in opposition with Lavie and Driver's (1996) proposal, object-based effects are impervious to modulation by the spatial setting of attention.

\section{REFERENCES}

AtChley, P., \& Kramer, A. F. (1998, November). Object-based attentional selection in three-dimensional space. Paper presented at the 39th Annual Meeting of the Psychonomic Society, Dallas.

BayLis, G. C., \& Driver, J. (1992). Visual parsing and response competition: The effect of grouping factors. Perception \& Psychophysics, 51, 145-162.

BERRY, G., \& KLEIN, R. (1993). Does motion-induced grouping modulate the flanker compatibility effect? A failure to replicate Driver \& Baylis. Canadian Journal of Experimental Psychology, 47, 714-729.

CHEN, Z. (1998). Switching attention within and between objects: The role of subjective organization. Canadian Journal of Experimental Psychology, 52, 7-16.

Driver, J., \& BAYLIS, G. C. (1989). Movement and visual attention: The spotlight metaphor breaks down. Journal of Experimental Psychology: Human Perception \& Performance, 15, 448-456.

DunCAN, J. (1984). Selective attention and the organization of visual information. Journal of Experimental Psychology: General, 113, 501-517.

Egeth, H. E., \& Yantis, S. (1997). Visual attention: Control, representation, and time course. Annual Review of Psychology, 48, 269-297.

EgLY, R., Driver, J., \& RAFAL, R. D. (1994). Shifting visual attention between objects and locations: Evidence from normal and parietal lesion subjects. Journal of Experimental Psychology: General, 123, 161-177.

ERIKSEN, C. W., \& HoffMan, J. E. (1973). The extent of processing of noise elements during selective encoding from visual displays. Perception \& Psychophysics, 14, 155-160.
Kramer, A. F., \& JACOBSON, A. (1991). Perceptual organization and focused attention: The role of objects and proximity in visual processing. Perception \& Psychophysics, 50, 267-284.

Kramer, A. F., Tham, M.-P., \& YeH, Y.-Y. (1991). Movement and focused attention: A failure to replicate. Perception \& Psychophysics, 50, 537-546.

Kramer, A. F., Weber, T. A., \& WATSON, S. E. (1997). Object-based attentional selection: Grouped arrays or spatially invariant representations? Journal of Experimental Psychology: General, 126, 3-13.

Lavie, N., \& Driver, J. (1996). On the spatial extent of attention in object-based visual selection. Perception \& Psychophysics, 58, 1238-1251.

MaCQuistan, A. D. (1997). Object-based allocation of visual attention in response to exogenous, but not endogenous, spatial precues. $P s y$ chonomic Bulletin \& Review, 4, 512-515.

Moore, C., Yantis, S., \& Vaughan, B. (1998). Object-based visual selction: Evidence from perceptual completion. Psychological Science, 9, 104-110.

NeELY, A., \& DaGENBACH, D. (1997). The effects of endogenous and exogenous orienting mechanisms on space-based vs. object-based organization. Unpublished manuscript.

Neely, A., Dagenbach, D., Thompson, R., \& CarR, T. H. (1998, November). Object-based visual attention: The spread of attention within objects and the movement of attention between objects. Paper presented at the 39th Annual Meeting of the Psychonomic Society, Dallas.

Treisman, A., Kahneman, D., \& Burkell, J. (1983). Perceptual objects and the cost of filtering. Perception \& Psychophysics, 33, 527-532.

VeCERA, S. P. (1994). Grouped locations and object-based attention: Comment on Egly, Driver, and Rafal (1994). Journal of Experimental Psychology: General, 123, 316-320.

VeCERA, S. P., \& FARAH, M. J. (1994). Does visual attention select objects or locations? Journal of Experimental Psychology: General, $123,146-160$.

WatSON, S. E., \& Kramer, A. F. (1999). Object-based visual selective attention and perceptual organization. Perception \& Psychophysics, 61, 31-49.

WeAVER, B., LuPiáñEz, J., \& WATSON, F. L. (1998). The effects of practice on object-based, location-based, and static-display inhibition of return. Perception \& Psychophysics, 60, 993-1003.

\section{NOTES}

1. The elements judged between objects and within objects were equivalent. The judgments required were intrinsically independent of objectbased discriminations. Eccentricity was kept equivalent across conditions.

2. Neely's communication of February 23, 1999.

3. Lavie and Driver did not specify the events that followed target display offset (e.g., whether the screen went blank until the fixation display of the next trial appeared and for how long).

4. In Lavie and Driver's experiment, subjects used the thumb to press " 0 " and the index finger to press " 2 ." During the practice block, the first 3 subjects complained that it was more comfortable for them to use the index and middle fingers instead. They switched to this response mapping after approximately 10 practice trials and continued to use it until the end of the experiment. The remaining 9 subjects received the instructions described in the Method section from the beginning of the experiment.

5. I thank Art Kramer and an anonymous reviewer for this suggestion.

(Manuscript received March 1, 1999; revision accepted for publication October $4,1999$. 